\title{
Aktuelle Ergebnisse zu Tanztherapie bei Schizophrenie und Autismus
}

\author{
Iris Bräuninger
}

In diesem Beitrag werden Ergebnisse zu zwei tanz-, bewegungstherapeutischen Interventionsbereichen vorgestellt: Schizophrenie und Autismus. Die Wirkung von Tanz-, Bewegungstherapie bei Menschen mit Schizophrenie wurde in einer aktuellen randomisierten Kontrollstudie untersucht. Fünf Arbeiten fokussierten auf das tanztherapeutische Potential bei Menschen mit Autismus, insbesondere Autismus-Spektrum-Störungen. Im DSM-5 und im ICD-11-Entwurf wird der mangelnden Unterscheidbarkeit zwischen frühkindlichem Autismus, Asperger-Syndrom, desintegrativen Störungen und tiefgreifenden Entwicklungsstörungen mit der Kategorie Autismus-Spektrum-Störungen (ASS) Rechnung getragen: Zwei Symptomgruppen bilden die Diagnosekriterien, nämlich qualitative Defizite in der sozialen Kommunikation und Interaktion und sich wiederholende Verhaltensmuster, Interessen oder Tätigkeiten, wobei das Auftrittsalter nicht mehr starr festgelegt ist (Sinzig 2015).

\section{Wirksamkeit von Tanz-, Bewegungs- therapie bei Schizophrenie}

Die Studie von Lee und KollegInnen (2015) untersuchte die Auswirkungen von Tanztherapie auf Affekte und psychotische Symptome bei schizophrenen PatientInnen. Die Teilnehmerlnnen ( $\mathrm{N}=38$, davon 20 Frauen) wurden zufällig auf die Tanztherapie-Gruppe $(n=18)$ und die Kontrollgruppe $(n=20)$ verteilt. Beide Gruppen erhielten medizinische Behandlungen. Die Tanztherapie-Gruppe wurde zwölfmal über einen Zeitraum von 60 Minuten angeboten. Jeweils vier Termine fokussierten auf Selbstwahrnehmung, interpersonelle Beziehungen und Beziehungen zwischen dem Einzelnen und der Gruppe. Die Tanztherapie-Gruppe zeigte keinen Effekt in Bezug auf die Angst-Symptomatik (STAI), jedoch reduzierten sich Wut (STAXI), Depression (BDI) und nega- tive Syndrome bei Schizophrenie (PANSS), und die Kontrolle über Wut (STAXI) steigerte sich zum Ende der Behandlung im Vergleich zur Kontrollgruppe.

\section{Wirksamkeit von Tanz-, Bewegungs- therapie bei Menschen mit Autismus}

Eine Machbarkeitsstudie von Koch und KollegInnen (2015) untersuchte die Auswirkungen einer siebenmal stattfindenden 60-minütigen Tanz-, Bewegungstherapie-Gruppenintervention bei $31 \mathrm{Er}$ wachsenen mit ASS, 23 davon Männer. Die Teilnehmerlnnen waren zwischen 16 und 47 Jahren mit einem Durchschnittsalter von 22 Jahren $(S D=7.7)$. Der Interventionsschwerpunkt lag auf dem Spiegeln von Bewegungen. Da eine Randomisierung nicht möglich war, wurden die Teilnehmerlnnen entsprechend dem Alter, Geschlecht und der Diagnoseschwere vergleichbar auf Behandlungsgruppe $(n=16)$ und Kontrollgruppe $(n=15)$ verteilt. Die Hypothesen, dass Tanz-, Bewegungstherapie psychisches Wohlbefinden (Hypothese/H1), Körperwahrnehmung $\left(\mathrm{H}_{2}\right)$, Unterscheidung zwischen dem Selbst und anderen $\left(\mathrm{H}_{3}\right)$, Empathie $\left(\mathrm{H}_{4}\right)$ und Sozialkompetenzen $\left(\mathrm{H}_{5}\right)$ verbessere, wurde mit Fragenbögen bei der Vor- und Nachuntersuchung überprüft, also vor Beginn der ersten und am Ende der siebten Stunde. Zusätzlich wurden Spiegelungsphänomene und qualitative Faktoren beobachtet und analysiert. Die Interventionen wurden in jeder Stunde wiederholt und setzten sich aus folgenden Teilen zusammen: a. Warm-Up im ChaceKreis, b. Führen-Folgen in Bewegungsdyaden, c. Baum-Kreis mit Führen durch Einzelne und Folgen der Gruppe und d. verbaler Abschluss. Die Hypothesen 1-3 und 5 wurden bestätigt, d.h. die Ergebnisse zu Wohlbefinden, Körperwahrnehmung, Unterscheidung zwischen dem Selbst und anderen und Sozialkompetenzen waren in der Tanzthera- 
piegruppe bei der Nachuntersuchung im Vergleich zur Kontrollgruppe signifikant besser. Die Ergebnisse zur Empathie zeigten keine signifikante Verbesserung. Ebenso wurde bei den Spiegelungsphänomenen keine Zunahme der Gegenbewegung beobachtet, welche als Indikator für Perspektivenübernahme gilt.

Eine qualitative Fallstudie von Edwards (2015) untersuchte, welche Sinneserfahrungen drei Männer und eine Frau mit Autismus (ASS und Asperger) im Rahmen einer achtwöchigen Tanz-, Bewegungspsychotherapie-Gruppe sammelten und auf welche Weise diese Erfahrungen deren Beziehungen und Bindungsverhalten formten. Eine 12-monatige Teilnahme in der Gruppe ging der Studie voraus. Es wurde eine qualitative, reflektierende TeilnehmerBeobachtungsmethodik gewählt. Zur Triangulierung erfassten zwei Therapeutlnnen systematisch ihre Bewegungsbeobachtungen und erstellten ein Reflective Journal (Edwards 2015, 10). Zum Ende jeder Stunde wurde die verbale Rückmeldung der Teilnehmerlnnen notiert. Die Ergebnisse wiesen darauf hin, dass die Anpassung an sinnliche Differenzierungen die Teilnehmerlnnen darin beeinflussten, wie sie Beziehungen und Interaktionen gestalteten. Diese erlernte Fähigkeit zu Accomodation und Adjustment könnte ein Hinweis darauf sein, dass Tanz-, Bewegungstherapie dazu beitrage, sensorische Empfindsamkeit und Beziehungen zu verbessern.

Wadsworth und Hackett (2014) integrierten in einer Einzelfallstudie über Tanz-, Bewegungspsychotherapie mit einem 23-jähirgen Mann mit ASS eine strukturierte Erzählweise in Form einer sechsteiligen Geschichte. Sie wählten einen praxisorientierten Forschungsansatz. Die Therapeutin hielt am Ende aller sieben Stunden ihre Beobachtungen auf einer kreativen Bewertungsskala fest, ebenso wie die Wahl der emotionalen Symbolbilder des Klienten zu Beginn und am Ende jeder Stunde. Die Autorlnnen schlussfolgerten, dass es sinnvoll wäre, in die Arbeit mit Erwachsenen mit ASS strukturierte Erzählansätze zu integrieren.

\section{Tanz-, Bewegungstherapie-Interventionen bei kindlichem Autismus}

Die Literaturübersicht von Scharoun und Kolleginnen (2015) beleuchtete die Auswirkung und den Nutzen von Tanz-, Bewegungstherapie bei Kindern mit ASS. Basierend auf Fallbeschreibungen im Einzel- und Gruppensetting präsentierten sie eine Vielzahl von tanztherapeutischen Interventionen und wiesen auf die Bedeutung synchronen Bewegens und von Spiegeltechniken hin. Bislang bestünde kein einheitliches Bild, weder über Vorzüge des Therapie-Settings (Einzel versus Gruppe), noch über wirksame Interventionen. Zukünftige Forschungsprojekte könnten die Wirksamkeit von kreativer Bewegung und Tanz als praktikable Option für Kinder mit ASS überprüfen.

Martin (2014) erforschte in einer theoretischen Literaturarbeit, wie Tanz-, Bewegungstherapie zur Förderung früher Entwicklungszusammenhänge zwischen sozialen und kommunikativen Aufgaben und motorischer Reifung bei kleinen Kindern mit ASS eingesetzt werden könnte. Ein tanz-, bewegungstherapeutischer Rahmen wurde vorgeschlagen, welcher auf die Stärkung der Körperwahrnehmung, motorischer Koordination und des Timings fokussiere und auf Interventionen wie Spiegelung, Körperbewusstsein und Rhythmus setze.

\section{Schlussfolgerung}

Die Studie zu Tanz-, Bewegungstherapie mit schizophrenen PatientInnen ergab signifikante positive Resultate in Bezug auf Affekt und psychotische Symptome. Die Forschungen zu Autismus wiesen auf die Leistungen und Grenzen bekannter Interventionen wie Spiegeln und Rhythmusarbeit hin und diskutierten die Integration neuer Techniken in die Tanz-, Bewegungstherapie. Die Ergebnisse zur Wirksamkeit der Tanztherapie bei ASS, um Wohlbefinden, Körperwahrnehmung, Unterscheidung zwischen dem Selbst und anderen und Sozialkompetenzen zu verbessern, sind vielversprechend, wie die Ergebnisse der Studie von Koch und Kolleginnen (2015) zeigten. Die Notwen- 
digkeit zukünftiger Forschungsprojekte mit gröBeren Fallzahlen wurde bei mehreren Autorlnnen thematisiert.

\section{Literatur}

Edwards, J. (2015): Exploring sensory sensitivities and relationships during group dance movement psychotherapy for adults with autism. Body, Movement and Dance in Psychotherapy 10 (1), 5-20, http://dx.doi.org/10.1080/17432979.2014.978894

Koch, S. C., Mehl, L., Sobanski, E., Sieber, M., Fuchs, T. (2015): Fixing the mirrors: A feasibility study of the effects of dance movement therapy on young adults with autism spectrum disorder. Autism 19 (3), 338-350, http://dx.doi.org/10.1177/ 1362361314522353

Lee, H. J., Jang, S. H., Lee, S. Y., Hwang, K. S. (2015): Effectiveness of dance / movement therapy on affect and psychotic symptoms in patients with schizophrenia. The Arts in Psychotherapy 45, 64-68, http://dx.doi.org/10.1016/j.aip.2015.07.003
Martin, M. (2014): Moving on the spectrum: Dance/ movement therapy as a potential early intervention tool for children with Autism Spectrum Disorders. The Arts in Psychotherapy 41 (5), 545-553, http://dx.doi.org/10.1016/j.aip.2014.10.003

Scharoun, S.M., Reinders, N. J., Bryden, P.J., Fletcher, P.C. (2014): Dance / movement therapy as an intervention for children with autism spectrum disorders. American Journal of Dance Therapy 36 (2), 209-228, http://dx.doi.org/10.1007/s10465-0149179-0

Sinzig, J. (2015): Autismus-Spektrum-Störungen. Monatsschrift Kinderheilkunde 163 (7), 673-680, http://dx.doi.org/10.1007/so0112-014-3242-2

Wadsworth, J., Hackett, S. (2014): Dance movement psychotherapy with an adult with autistic spectrum disorder: An observational single-case study. Body, Movement and Dance in Psychotherapy 9 (2), 59-73, http://dx.doi.org/10.1080/17432979.2 014.893259

\section{Die Autorin}

\section{Dr. Iris Bräuninger}

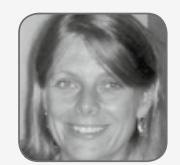

Wissenschaftliche Mitarbeiterin

Psychiatrische Universitätsklinik

Zürich, Dozentin im Masterstudiengang Tanztherapie an der Autonomen Universität Barcelona (UAB), Supervisorin, Ausbilderin und Lehrtherapeutin der deutschen und spanischen Berufsverbände (BTD, ADMTE), Kestenberg Bewegungsnotatorin, Psychotherapie (ECP), Private Praxis für Supervision und Therapie

\author{
Dr. Iris Bräuninger \\ Wissenschaftliche Mitarbeiterin \\ Psychiatrische Universitätsklinik Zürich \\ Direktion Pflege, Therapien und Soziale Arbeit \\ Forschung und Entwicklung \\ Lenggstr. 31 | $\mathrm{CH}-8032$ Zürich \\ Tel. 0041-(0)443842717 \\ 0041-(0)774422676 \\ iris.braeuninger@puk.zh.ch oder \\ dancetherapy@mac.com
}

